\title{
Statistische Daten grafisch darstellen
}

\author{
WISSENSCHAFT ERKLÄRT Um Messungen und statistische Berechnungen \\ schnell überblicken zu können, empfiehlt es sich, die Ergebnisse grafisch \\ darzustellen. Welche Werte sich wie abbilden lassen, beschreibt dieser Artikel.
}

S

tatistische Berechnungen beruhen auf

verschiedenen Bedingungen: Eine Messbedingung ist zum Beispiel, dass man bei intervallskalierten Messungen den Mittelwert berechnen und nutzen sollte ( $\odot$ physiopraxis 2/08, S. 20, „Wissenschaft erklärt: Beschreibende Statistik Teil 1“). Nicht immer ist der Mittelwert aber aussagefähig. Beispielsweise wäre es kaum sinnvoll, den Mittelwert einer Haarfarbe zu berechnen. Haarfarben lassen sich hingegen gut mit einem sogenannten Flächendiagramm grafisch darstellen.

Ein gutes Beispiel aus der Physiotherapie ist, den Anteil weiblicher und männlicher Personen in einer Praxis oder den Anteil akuter und subakuter Patienten in der Therapie grafisch abzubilden. Eine grafische Darstellung solcher Anteile oder Kategorien erfolgt am besten mit sogenannten Kuchendiagrammen, wie man sie kennt von der Sitzverteilung im Bundestag bei Wahlergebnissen.

Ergebnisse, die ordinalskaliert sind, wie beispielsweise die Zufriedenheit mit einer physiotherapeutischen Behandlung (sehr hohe Zufriedenheit bis sehr niedrige Zufriedenheit) oder die Anzahl Kinder in einer Familie, lassen sich in einem Säulen- beziehungsweise Stabdiagramm darstellen.

\section{Histogramme liefern einen Überblick >} Für Darstellungen wie die der Altersstruktur der Patienten einer Praxis eignen sich sogenannte Histogramme. Sie zeigen, wie häufig bestimmte Werte vorkommen, und liefern deshalb eine sehr gute Übersicht über die Altersverteilungen oder auch über die Krankheitsdauer von Patienten (॰ Abb. 1). Man könnte mithilfe eines Histogramms die Frage beantworten, ob es mehr jüngere oder mehr ältere Patienten beziehungsweise mehr chronische als akute Patienten in der Praxis gibt.
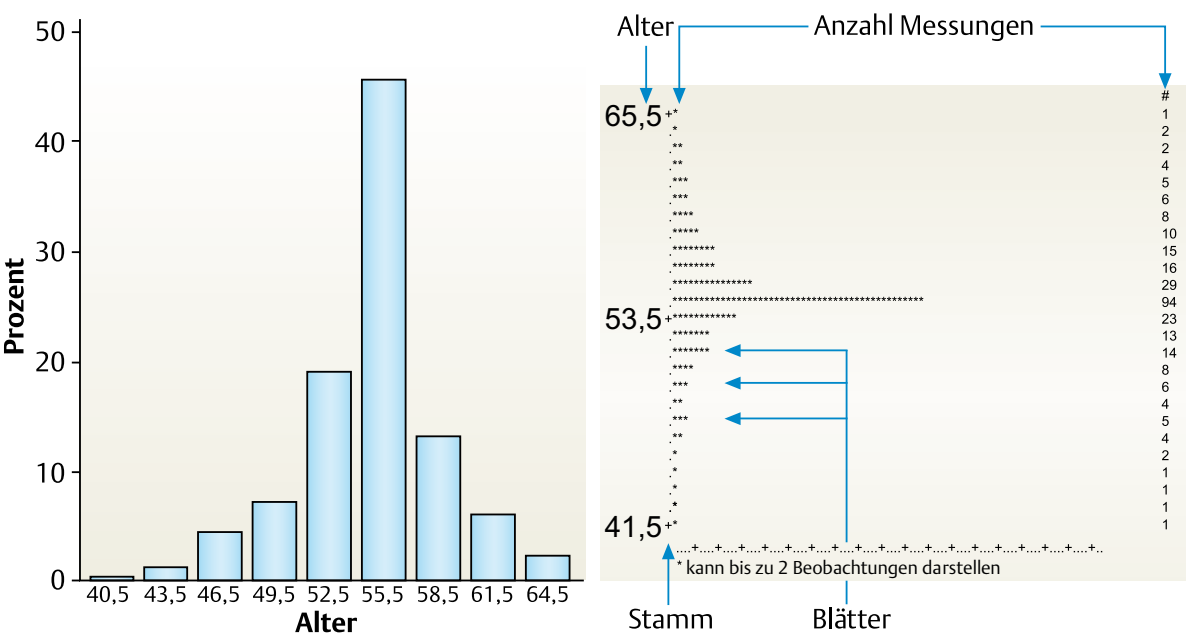

Abb. 1 Histogramm zur Altersverteilung

Abb. 2 Alternative: eine Stamm-Blatt-Grafik

Eine andere Art Histogramm ist die sogenannte Stamm-Blatt-Grafik (॰ Abb. 2), oder auch Stamm-Blatt-Diagramm genannt (engl.: steam-and-leaf-plot oder stemplot). Ähnlich wie bei einem Histogramm oder einem Boxplot werden in Stamm-Blatt-Abbildungen Daten visualisiert. Statistische Werte wie Modalwert, Median und Quantile lassen sich hier optimal ablesen. Einen Nachteil hat eine Stamm-Blatt-Grafik jedoch: Die Darstellungsform ist bei größeren Datenmengen nicht so gut geeignet wie klassische Histogramme.

\section{Histogramm: Verteilungen erkennen >} Ein Stamm-Blatt-Diagramm hat zwei oder drei Spalten. Die erste Spalte ist der Stamm (zum Beispiel das Alter von 20 bis 70 Jahren), die zweite Spalte enthält die Blätter (Anzahl an Patienten im jeweiligen Alter). Eine eventuell vorhandene dritte Spalte erspart das Zählen der Blätter und stellt die Häufigkeit (Anzahl der Patienten) als Zahl dar. Erstellt man regel- mäßig, also beispielsweise monatlich oder halbjährig, Histogramme, lassen sich diese miteinander vergleichen. Der Vergleich gibt Hinweise darauf, ob und wie stark sich dargestellte Anteile verändern. Allgemein kann man sagen: Histogramme und Stamm-Blatt-Grafiken geben Aufschluss über Verteilungen. Wohl eine der bekanntesten Verteilungen ist die sogenannte Gauß'sche Glockenkurve. Sie entspricht der Normalverteilung und war sogar auf den 10-Mark-Scheinen abgebildet.

Fazit > Grafische Darstellungen wie zum Beispiel Histogramme oder Stamm-Blatt-Grafiken liefern Informationen über die Verteilung erhobener Daten. Dies ist besonders für Überlegungen zum statistischen Testen wichtig, nämlich dann, wenn man Ergebnisse einer Patientengruppe vor und nach einer Behandlung vergleichen möchte. Wie man Messergebnisse vergleichen kann, lesen Sie in der nächsten Ausgabe.

Prof. Dr. Jan Mehrholz 